\title{
In Service to Others: A New Evolutionary Perspective on Human Enhancement
}

\author{
Hugh Desmond \\ IHPST (CNRS/Paris I-Sorbonne) \\ University of Antwerp
}

Forthcoming in The Hastings Center Report

\begin{abstract}
In enhancement ethics, evolutionary theory has been largely perceived as supporting liberal views on enhancement, where decisions to enhance are predominantly regulated by the principle of individual autonomy. In this paper I critique this perception in light of recent scientific developments. Cultural evolutionary theory suggests a picture where individual interests are entangled with community interests, and this undermines the applicability of the principle of autonomy. This is particularly relevant for enhancement ethics, given how - I argue - decisions to enhance are often influenced by desires to increase social status. The "service view on enhancement", based on principles of service and trust, is proposed as offering better guidance for the challenges of social living.

Keywords: ENHANCEMENT - LIBERALISM - EVOLUTION - AUTONOMY Social STATUS - TRUST - SERVICE
\end{abstract}

\section{Introduction}

Although science may be neither necessary nor sufficient for ethical argumentation, evolutionary theory has played a prominent role in recent enhancement ethics. For instance, claims that enhancement should be constrained by a fixed human nature or by 
givenness seem difficult to hold given how the human species evolved and continues to evolve. ${ }^{1}$

Another evolutionary argument that seems to have gained particular currency appears to offer a direct, positive argument for enhancement. It appeals to the fact that humans evolved in hunter-gatherer environments but live in cosmopolitan and urbanized environments with which they are mismatched. This is a major hypothesis in evolutionary psychology and psychiatry, and is linked, for instance, to the widespread prevalence of some mental health disorders. ${ }^{2}$ In the context of enhancement ethics, the mismatch hypothesis is leveraged to argue that humans are so deeply maladapted, unable to rise to the challenges of cosmopolitan and multicultural environments, that only technological enhancement can ensure that humans are fit for the future. Hence many legal and institutional barriers constricting enhancement should be lifted, so that enhancements can be used in the quest for personal well-being. 3

It is striking how, through such arguments, evolutionary theory has broadly been perceived as supporting liberal views on enhancement, where decisions on whether to enhance or not are predominantly guided by the principle of individual autonomy. And while liberal views can range from the social liberal to the libertarian, in enhancement ethics, the center of gravity in recent literature has tended towards the latter. According to libertarian views, enhancements are to be judged as Millian "experiments in living": as long as they do not actively harm others, they are ethically commendable expressions of individual autonomy. 4

A libertarian view on enhancement may not convince everybody, but it is not always easy to show where precisely the view goes wrong. For once one accepts that humans are deeply maladapted, it is very difficult to escape the logic of utilitarian reasoning, which leads inexorably to the conclusion of liberalizing enhancement in one way or another, if not through legal and institutional deregulation (the libertarian conclusion), then through fair, institution-backed administrations of enhancement (the social liberal conclusion). 5

My aim in this article is to undermine the starting point in this reasoning by drawing on cultural evolutionary theory. This shows how the role of the social and cultural environments has been unjustifiedly ignored or downplayed in recent enhancement ethics. The implication is an undermining of the fundamental presumption of liberal 
views: a relatively strong distinction between the interests and decisions of the individual, and those of the community. It is just this distinction that is undermined given what we know about how our species evolved. As a very social species, our desires are significantly shaped by the norms and patterns of behavior of the community we inhabit. Our desires are not quite "our own".

One very concrete dynamic that illustrates this intertwining of individual and community is competition for social status. The desire for status, as we will see later, is fundamental motivator for humans, regardless of culture, gender, age, or even personality. Unsurprisingly, this motivator also appears across empirical studies on attitudes towards enhancement. From height enhancements to performance enhancing drugs and cognitive enhancement, decisions to enhance are often motivated by desires to increase social status - potentially leading to perverse status competition.

This dynamic does not so much falsify liberal views as show them to be incomplete. I argue that neither the principle of no harm nor that of distributive justice gives satisfactory guidance to individuals navigating such status competition. Deliberations on whether to enhance thus require ethical resources not found among liberal views.

What could be more promising instead? I propose the service view on enhancement, in which the dominant principle guiding individual decision making is not autonomy but service: not the "own" good and wellbeing in the strict sense, but the good and wellbeing of others and that of the community at large. This view is, I argue, more grounded on what we know about human evolution, and is also more in tune with the real concerns, anxieties, or competitive urges that members of communities must respond to.

\section{Evolution and Liberal Views of Enhancement}

Enhancement ethics is future oriented. It is about what humans might become. Unsurprisingly, therefore, it has long been inspired by the science of what humans have been in the past-namely, evolutionary science. The old eugenics was strongly inspired by the $19^{\text {th }}$ century understanding of the theory of natural selection; ${ }^{6}$ similarly, today's enhancement ethics draws on two common evolutionary arguments. The first argument turns on the fact that evolution debunks (folk-psychological) essentialist understandings of human nature. 7 There are simply no universal, unchanging properties that characterize 
all members of the Homo sapiens. Hence enhancement technology is not changing anything that might not change anyway. Beliefs in (essentialist) human nature are ungrounded and should not constrain enhancement. ${ }^{8}$ Is this debunking of human nature definitive? Not necessarily. In the past decades there have been some attempts to resuscitate human nature as a scientifically legitimate concept.9 However, the resulting concepts of human nature are causal or descriptive concepts, void of moral significance. ${ }^{10}$

A similar argument has also been applied to the concept of "givenness". ${ }^{11}$ After all, humans have been technologically enhancing their "given" capacities since the dawn of the Homo genus about two million years ago. Our evolutionary history paints us as enterprising, autonomous individuals; there is no natural or given state of affairs to constrain human autonomy. Human enhancement is business as usual.

If the first evolutionary argument supports the right to enhance, the second evolutionary argument - and the main focus of this paper - supports to duty to enhance. In brief: the vast majority of the evolution of the Homo genus (i.e., about $99.5 \%$ of the past 2 million years) took place in an environment of small-scale hunter-gatherer societies, and today human traits and human cognition remain designed for the specific challenges of that ancestral environment. This hypothesis has been used to great success in evolutionary psychology to explain common mate preferences, sexual taboos, and emotions, ${ }^{12}$ and why some mental disorders, such as major depressive disorder or attention deficit disorder, seem to be on the rise. ${ }^{13}$ This hypothesis has been used in enhancement ethics to provide an argument in favor of enhancement - or, at least, for enhancement of any trait that is not optimally adapted to the challenges of our urbanized, anonymous, and increasingly online living.

Here is one concrete instance of this mismatch argument:

After all, our brains are products of evolution, which is a blind process that hardly seeks to maximize the good, or make us morally best. Evolution 'cares' only about reproductive success. Moreover, even if the evolutionary process somehow led to what is in one sense an optimal result, this result may be optimal only in the environment in which our very distant ancestors lived. It is very unlikely to be optimal in our utterly different modern environment. (There was, for example, no police in the primeval savannas, nor were there planes or hijackers ... $)^{14}$ 
Other examples could be cited, ${ }^{15}$ and variations on this positive evolutionary argument have been widely adopted by prominent ethicists. ${ }^{16}$

The mismatch argument works in part through its rhetorical force. One barrier to widespread acceptance of enhancements progress might lie in our evolved cognitive biases, such as biases towards the status quo, ${ }^{17}$ somewhat similar to how the eighteenthcentury philosopher Nicolas de Condorcet thought the barriers to human progress lay in "ignorance" and the "yoke" of political or religious authority. ${ }^{18}$ The mismatch argument creates the impression that liberal views of enhancement are situated within a larger family of liberal views that go back to the Enlightenment. The rhetorics of this association lies in the suggestion that, also today, the liberalization of enhancement must overcome similar trials in human obscurantism and irrationality.

In terms of its logic, the mismatch argument seems to work in two ways. First, it shifts the utilitarian calculus in favor of enhancement: the current state of human phenotypes is very maladapted, and this maladaptedness is engrained in our genetic makeup, and hence the potential benefits from enhancement are very large. In other words, the argument supports "enthusiasm" for enhancement, where the relative benefits of enhancement are emphasized. ${ }^{19}$ However, such a utilitarian calculus could also be used to justify a collectivist approach to enhancement, so the maladaptedness argument must do more than simply shift the utilitarian calculus in favor of a liberal (and specifically libertarian) view on enhancement. (Note that the argument for enthusiasm works best if human phenotypes are suboptimal, but not too suboptimal; if our desires and volitions are too deeply maladaptive, then the principle of individual autonomy is eroded and a societal decision to let the decision be made by hard data and algorithms will compel us to accept enhancement, perhaps even the radical enhancement of transhumanism. ${ }^{20}$ )

A second and more fundamental way in which the maladaptedness argument promotes a liberal view perhaps lies in how it paints a direct, simple, and intuitive view of humans and human evolution as "individual-centered". In such a individual-centered view, adaptiveness is spelled out in terms of capacities of the individual - not social or moral norms, or social structures. The unit of selection is the individual, and the only processes considered are those concerning the individual and the individual's direct environment. 
The individual-centered view does not explicitly reject group selection or cultural evolution; it just ignores it. It is perhaps best understood as a certain interpretation of the evolutionary psychology of the 1980 os and $1990 \mathrm{os},{ }^{21}$ and fits in well with approaches to defining well-being in terms of individual capacities and changes in well-being in terms of changes in "the biology or psychology of a person"22. This focus on the individual comes under pressure when we consider how entangled individual interests are with community interests.

\section{Social Status and the Decision to Enhance}

When a human and chimpanzee toddler are given the same spatial or causal task to solve, they do about as well (or rather, poorly). However, when it comes to social tasks, like imitating the human experimenter's solution of following their gaze, then the human toddler vastly outperforms the young chimpanzee. ${ }^{23}$ This phenomenon of imitation and social learning is the crucial feature of cultural evolutionary theory's view on human evolution. ${ }^{24}$ The human evolutionary story is a story of social learning, in which knowledge and skills are transmitted from one individual to the next and from one generation to the next via social processes. ${ }^{25}$ Social learning is thought to be central for the dramatic geographic expansion and population growth of the human species. ${ }^{26}$

This gives rise to what can be called the "group-centered view" of human evolution, in which the community or group is the central unit of selection, adapting to changes in the external environment and undergoing selective competition. Individuals develop in social-cultural environments, and the structure of the latter changes over time: new behavioral habits, moral norms, and technological know-how. The community affects individual reasoning and valying, which in turn affect social norms, and in this way, our actions in the private sphere - undertaken in our "experiments of living" - are protopublic acts with ripple effects throughout the community.

Social Status Norms. I will be arguing that one type of social norm in particular - status norms - seems to be highly relevant for understanding decisions to enhance. For this it is helpful to have some background in evolutionary perspectives on status, because the term "status" may evoke predominantly negative connotations today (oppression,

privilege, structural violence, and so on). From an evolutionary perspective, "status 
differences" refers to ways of structuring a group of organisms so that intragroup conflict about access to resources is minimized. For example, the pecking order among chickens allows a group of chickens to work out which individual may peck at food first, which individual second, and so on, without physical conflict. Such hierarchies are widespread among mammals, birds, fish, as well as invertebrates 27 - and humans.

The main difference between the human and other animal species lies in how human status hierarchies are determined not only by "dominance," or the threat of force, but also by "prestige," which is freely given to individuals in recognition of a high degree of competence in some activity. ${ }^{28}$ So humans have evolved ways of shaping status hierarchies so that status is assigned to the persons whom others can learn the most from, and also who might likely make the best decisions for the group. Status competition, as long as it is for prestige, need not be zero-sum, as it can lead to better services being rendered to the community. Prestige represents a prosocial way of organizing status hierarchies.

However, like any moral innovation, it can be hijacked. High-status individuals hold sway over large swathes of the community, and hence at some later time they can, in principle, convert their social networks into coalitions to suppress a rival. ${ }^{29}$ Egotistic dominance behaviors lurk around the corner, even if status was originally freely conferred to the individual. Hence human societies are characterized by "service-for-prestige" norms: high-status individuals are expected to provide some form of service (expertise, risk, judgment, time) in exchange for prestige, and are punished if they fall afoul of the norms. ${ }^{30}$

In this way, most if not all human cultures are characterized by a constellation of service-for-prestige norms - something that I will dub the "ethos of service" for purposes here. In reality, this ethos is, of course, not evenly distributed, and some communities have a weaker ethos of service than others. For instance, apparently the "dark triad" of dominance-related personality traits (Machiavellianism, narcissism, psychopathy) is significantly more prevalent among corporate and political leaders compared to the general population. ${ }^{31}$ Nonetheless, it is not wrong to deem an ethos of service as the norm for human populations. As suggested by very term "dark triad", the ethos of service is still held as a normative ideal even if it may not be realized. 
Social Status and the Decision to Enhance. Imagine an enhancement that has as little to do as possible with social status - perhaps a hypothetical drug that allows one to blink "abnormally" fast, beyond species-typical limits. Why is there no industry researching and investing in breakthroughs for blinking drugs? Why are lawmakers and ethicists not debating the acceptability of such enhancements? Because nobody cares: nothing is at stake. Perhaps some biohacker might find some idiosyncratic interest in such enhancements, but it is hard to imagine such an enhancement being of any public interest. 32

Conversely, some of the most ethically controversial classes of enhancement directly or indirectly involve competition for status. The most straightforward example is height enhancement. Like almost all other animal species, humans unconsciously assign higher status to tall or large individuals. 33 This bias shows up in various statistics, for instance in correlations between height and military or corporate rank, or between height and income (Blaker et al. 2013). It also shows up in experimental psychology, with most marked effects on the judgment of males: taller males are perceived as possessing superior leadership, intelligence, and even health. 34

This privileging of height, contrary to appearances, is not merely a quirky vestige of our evolutionary past. In particular, it shows up in clinical studies and the ethics of "idiopathic short stature" - the condition of short stature without any underlying pathology. Some parents (consciously or not) view short stature as a "disadvantage in [the] child's social life and career" or even as a "disability".35 Perhaps in recognition of these lived realities, the U.S. Food and Drug Administration sanctions human growth hormone therapy when the child is in approximately the first percentile for height, even though the child may not be suffering from a growth hormone deficiency.

What matters here is relative height, not absolute height. In fact, what has counted as "short" has fluctuated considerably over history, ${ }^{36}$ and the competition for relative height is an obvious instance of a zero-sum competition. So, to briefly anticipate the consequences for liberal views, one can already wonder to what extent parents are making an autonomous decision when they opt for human growth hormone therapy for a short child even if perfectly healthy. The parents are not being forced to enhance, but at the same time their calculus is driven by fears that their child will have to face bullying, being passed over for jobs and promotion, and so on. ${ }^{37}$ The decision is not "coerced" in any hard 
sense, but the norms of the social-cultural environment are setting the parameters for the parents' calculus, and one can only assume that most parents would decide differently if the costs of short stature were not so steep.

A similar story can be told of performance enhancement. In a recent empirical study of the motivations of athletes to take performance enhancers, $3^{8}$ the most widespread motivations pertained directly athletic performance, even when it came at a cost to personal health. Why is athletic excellence desired? The researchers document that there are many second-order motivations that have to do social status: improving one's social image, securing respect from others, gaining friends, obtaining what the researchers term "hero status", getting more media attention, and acquiring financial gains. Once again, status norms in the social-cultural environment are setting the parameters for the decision whether to enhance.

Some types of enhancement undertaken for aesthetic reasons may also have second-order motivations related to status. For instance, when anabolic steroids are used as a cosmetic enhancement to increase muscle mass, users point to aesthetic reasons and the desire for "enhanced confidence". 39 But why should having a muscular body be aesthetic or justify self-confidence? Basic evolutionary psychology tells us here that humans about muscle mass (at least in males) because, as a way of increasing one's physical formidability, it increases perceived dominance status..$^{40}$

How deep is the link between motivations for enhancement and social status? The rationale for the link between enhancement and status is simply that an enhancement of some desirable capacity $\mathrm{X}$ can be used to either gain a competitive advantage over others with regards to $\mathrm{X}$ (in a competition for dominance) or to provide a better service to the community through $\mathrm{X}$ (and hence increased prestige). The capacity $\mathrm{X}$ need not be used in status competition; however, the reality is that social status represents one of the fundamental motivators for humans. In their review study, Cameron Anderson et al. even conclude that our evolved desire for status is a "universal" human motivator: it is discerned across cultures, genders, ages, and personalities. ${ }^{41}$ Given the centrality of the status motivation, and given how enhancements can be co-opted for status competition, one should not be surprised that motivations to enhance are often even be subordinated to status-related motivations. 
One could wonder whether the observation holds for all types of enhancements. This depends in part on the definition of "enhancement". Health-related interventions do not seem to have much to do with status: vaccinations stimulate adaptive immunity, and glasses correct for myopia. Whether such interventions should be categorized as enhancement or not is subject to disagreement: some would wish to preserve some distinction between "therapy" and "enhancement". ${ }^{2}$ Relationship and love enhancements also would not seem to be motivated by status, but again, it's debatable whether love drugs aim beyond species-typical norms or merely at achieving what is typically considered "healthy." 43

Despite these questions, it is safe to say that status seems central to the motivations for at least some types of enhancement technology. In such cases, given how individual interests are entangled with status norms (in which community interests are represented), the mismatch argument for enhancement seems much less plausible. Apparent mismatch may be the product of a suboptimal social and cultural environment - and wayward status hierarchies in particular. which may value properties such as "being taller than most others" or being more muscular or having shorter feet. The appropriate response is not enhancement, in order to better adapt to the environment, but rather social action to change the environment.

This line of thought also casts doubt on the basic libertarian normative idea that as long as nobody else is harmed by an enhancement, a person should be free to undergo enhancement in any way they see fit. The problem is not that the libertarian idea is wrong but that it is fatally incomplete. It does not recognize that individuals desire some enhancements because they would improve status, and it does not consider that narrowly pursuing status can lead to zero-sum status competitions in which everyone is left worse off. The basic libertarian view has a blind spot about perverse status competition.

\section{Liberal Responses: No Harm and Justice}

The liberal approach to enhancement could be defended against this critique. The condition that enhancements must not harm others and a requirement for distributive justice, for example, might allow for liberal views to put limits on enhancements that are 
aimed at gaining dominance status. However, the entanglement of individual and community interests turns out to be fundamentally problematic for any liberal view.

Avoiding harm through moral enhancement. The first defense is the threat from perverse status competition constitutes a form of harm, and hence can motivate restrictions on individual autonomy. In fact, our unfortunate, status-oriented moral psychology could be taken as a reason for carrying out moral enhancement, in order to suppress our desires for dominance and promote our desires for service. Thus, a liberal proponent of enhancement might argue, the solution lies not in less enhancement but in more and better-targeted enhancement.

Usually, moral enhancement is discussed as a potential response to exclusivist tendencies within our moral psychology: we tend to be negatively biased towards humans who do not belong to our "group", defined by culture or ethnicity.44 The defense here applies similar considerations to the distinction between dominance and prestige status. Just as inclusivist morality has considerable precedent in human evolution, so do servicefor-prestige norms; even so, it could be argued that our concern for the latter could use a "boost by biomedical means".45 Perhaps prosocial drugs could raise levels of prosocial hormones or neurotransmitters like oxytocin or serotonin, with the effect that low-status individuals would not make decisions based on a desire of dominance and such that highprestige individuals would use their position to obtain power and impose their wishes.

Why this defense, upon reflection, does not seem attractive lies in the nature of the concept "service". What does it mean to "serve" others, rather than to "seek dominance"? There is sometimes a fine line between service and the pursuit of social dominance. If the committee chair silences someone during a meeting, then the chair is using their position of dominance. However, it is not primarily dominance if the act of silencing is done out of service to the group - for instance if the person was speaking hatefully. But it would be a pure dominance display if the participant was silenced merely for presenting a dissenting opinion. This raises the question: who decides what service means, and with it, what behaviors are to be judged as mere displays of dominance and what as pro-social behaviors?

Moreover, prosocial tendencies can end up supporting oppressive status norms. For instance, a moral reformer may be motivated by service and not by dominance, but may not be perceived as such by the majority if the reformer is viewed as "antisocial" and 
as flouting social norms. In this way, the notion of "community interests" is ambiguous. Does it have to do with respecting preferences of immediate peers, or those of future generations? This question goes beyond the scope of this article, but the fact it arises shows how the further strengthening of service-for-prestige norms cannot be automatically obtained by pharmaceutically enhancing prosocial hormones or neurotransmitters.

More ethically plausible, though less technologically plausible, would be a form of moral enhancement that directly improved moral decision-making - a chip or computerbrain interface, perhaps. At least in theory, such an enhancement could help with the type of fine-grained decision-making under uncertainty required to promote service-forprestige norms. However, once the moral enhancement becomes powerful enough to actually guide decision-making, then the question arises of who would design such a moral enhancement? Which values would be programmed into the chip, which ethical principles? This response to the problem of perverse status competition thus seems to lead us very far away from the core libertarian idea, and towards, at best, a paternalistic social liberalism. However, in that case, the designers would need to possess some prior understanding of service-for-prestige norms in order to design such moral enhancements. So, for at least a segment of the population, the problem of perverse competition would not be solvable by moral enhancement.

Distributive Justice. The second strategy is that of a social liberal who never would have subscribed to the libertarian idea in the first place: in this person's view, the danger of perverse competition is a familiar danger associated with positional goods and a strong rationale for regulation by public policy on grounds of distributive justice. ${ }^{46} \mathrm{In}$ the social liberal's view, the state and public institutions should step in to prevent the worst excesses of status competition and ensure a baseline of fair distribution of goods, so that basic rights and equality of opportunity are protected. Allen Buchanan and Russell Powell have recently argued that exclusionary tendencies in our moral psychology require correction through principles of distributive justice, 47 and their argument can be expanded to address the challenges from enhancements that confer social dominance.

In many important cases, this is the right response: when it is in the community's interest to have a fair distribution of "social and economic advantages" accruing to individuals, principles of distributive justice can help address the problems of individual 
autonomy that arise if enhancements conferred those advantages. $4^{8}$ However, the primary recipient of the advantages of enhancement is assumed here to be the individual. The community's interests in the enhancement are secondary. Sometimes, however, an enhancement might be considered primarily as a gift to the community, and in these cases, principles of justice that equally distribute the enhancement are inappropriate. This can be illustrated with two contrasting examples.

First, consider performance enhancing drugs. Do athletes take these to serve the community better? Interestingly, some have argued for that the use of enhancers by athletes is similar to the use of beta-blockers by classical musicians in that both kinds of drugs lead to better entertainment. 49 According to this argument, enhancers promote an artistic service to the community, , and not merely a narrowly individual good. However, this does not seem to be the best way of understanding performance enhancement. Social goods do not seem to figure much at all in the athletes' reasons for doping: they are more interested in achieving wealth or "hero status" ${ }^{\circ}$. In addition, the public seems to care more about relative performance than about absolute performance; we still celebrate winners even when records are not broken, and we enjoy youth and college leagues as much as professional leagues, even though the absolute performance may often be lower. Finally, the emphasis of sports-governing bodies on fairness ${ }^{1}$ in regulating doping use suggests that performance-enhancing drugs are viewed primarily as promoting narrowly individual goods.

A contrasting example lies in cognitive enhancement. In the literature on this topic, $5^{2}$ education is often seen as the touchstone of a morally acceptable cognitive enhancement. However, if we focus on college and graduate school education, we see how cognitive enhancement inserts itself at the very heart of status hierarchies. This is why third-level education is ethically ambiguous in a way that shows the limits of distributive justice.

Much academic education (consider law, medicine, psychology, or engineering) is directed toward entry into a profession. In fact, academia is often seen as an arena where rival professions carry out proxy conflicts over who can claim "expertise." 53 The close link between academia and high-status professions is one of the main reasons why educational attainment is so strongly positively correlated with life outcomes, whether one measures 
that according to subjective well-being, self-esteem, or mental health, ${ }^{54}$ or even chance of disease, and mortality from all causes. 55

Many parents today recognize that education is a positional good. It is well documented how certain parents are more actively involved so that their children will gain access to status-enhancing educational institutions.56 The most common motivations cited are concern for the child's future "well-being" and "success", where the latter specifically means success in the competition for status. ${ }^{57}$ Moreover, the scarcer education is and the fewer highly educated people there are, the more higher education contributes more to subjective well-being. $5^{8}$

Education, especially at a college level and beyond, sits uneasily on the dominanceprestige divide. The competence it engenders can lead to better services being rendered to the community, in which case the society as a whole benefits. However, education can also be pursued for the sake of enjoying the privileges and benefits associated with status. If the latter are not subordinated to service, then the privileges become means to allow one's individual preferences to weigh more strongly in the community: this is a subtle form of dominance.

This dual nature of education is sometimes overlooked in the cognitive enhancement literature, but it is not new. In ancient Greece, when systematic education was still a relatively new development in human history, sophists and philosophers alike were viewed with suspicion. Thus Isocrates (436-338 BC) was charged with "corrupting the youth": endowing them with rhetorical skill to gain an unfair advantage over peers during court trials or debates over policy. Socrates, of course, was charged and sentenced to death over the same "crime"; education, and the rhetorical and argumentative supremacy it afforded, was viewed with suspicion. Today, scientific experts and highly educated liberal elites are viewed with suspicion and distrust, and this distrust can be interpreted as reflecting a moral uncertainty: will the powerful cognitive enhancement that higher education represents be used for the benefit of the community, or to promote patterns of dominance?

The fine line between dominance and prestige, and between privilege and service that is strongly apparent in higher education, means that this form of cognitive enhancement is both an individual good as well as a social good. This makes it difficult to regulate the dangers of perverse competition through the conceptual framework of public 
policy and distributive justice. If we follow the latter, then tertiary education is conceptualized in terms of equality of opportunity. The problem is not that this is wrong, but that it is problematically incomplete: the main question is not who gets the powerful cognitive enhancement, but whether those who benefit from it use it for service or for dominance. Enhancement through tertiary education is not merely an individual good but also a social good.

This is why professional ethics offers a better model for thinking about how cognitive enhancements should be regulated, where individual interests and community interests are so strongly entangled. Associations of physicians, lawyers, or psychologists do not regulate the conduct of their members in the way the International Cycling Union regulates cyclists in the Tour de France. There is, in general, considerably more trust in practicing professionals, not only from governing bodies, but also from the clients and customers of the professionals. Moreover, attempts at coercing service-oriented attitudes from individual members, whether through nudges or punishment, are often selfdefeating. 59 This is why the self-regulation of professions is typically formulated more in terms of ethics rather than policy oriented towards justice: having an ethics oriented towards an ideal of service is, according to some sociologists, a defining characteristic of what professionalism means. ${ }^{60}$ Inequality is less of pressing issue: if a professional decides to undergo additional cognitive enhancement, they may indeed accrue further social status and privilege (as with the specialist physician who undergoes yet further specialization), but the expectation is that this increased competence will be put at the service of the community and that all will benefit.

In sum, it should be acknowledged that some enhancements predominantly entail individual privilege: when this is the case (e.g. performance enhancing drugs), then the principle of distributive justice is applicable. However, important classes of enhancement such as cognitive enhancement, are not merely individual goods but also social goods. They involve both prosocial responsibilities as well as privileges. For such types of enhancement, a redistribution according to fairness principles may not prevent perverse competition from happening anyway. Instead, the appropriate response lies in strengthening the ethics of those undergoing enhancement. This is the basic idea underlying the service view on enhancement. 


\section{The Service View of Enhancement}

Let me recap the argument so far. Recent scientific developments in cultural evolutionary theory suggest a group-centered view of human evolution where the interests of the individual and community are intertwined, especially visible in how communities are structured into status hierarchies which benefit both individuals and the community. Decisions to enhance are strongly influenced by status hierarchies and can lead to perverse competitions for dominance. This view casts doubt on the evolutionary rationale for the libertarian idea, namely that individuals need enhancement due to their deeply maladaptive evolved traits: the apparent mismatch may simply reflect their inability to adapt to social-cultural environments, and the fault may lie in wayward status hierarchies. It also casts doubt on the main libertarian idea that, unless direct harm is threatened, individuals should be left unperturbed to pursue their wellbeing as they see fit, and if that includes enhancing their capacities, then that should be endorsed.

We cannot enhance our way out of perverse status competition. Perhaps school bullies can be pharmaceutically enhanced to stop bullying short children; however, the proper application of such moral enhancement presupposes that there are parents with a prior understanding of what is ethically desirable is needed. However, on a societal scale, there are no such "parents", at least if one holds to libertarian view. A social liberal view may seem more promising, by means of the principle of distributive justice. However, the principle of justice is only applicable to individual goods - when the goods are social in nature, then fair distributions among individuals can no longer be the guiding principle.

Given how individuals are embedded in social networks characterized by status hierarchies, the proposal is that the real question lies not in how to maintain individual autonomy (either through negative liberty, no harm, or distributive justice), but rather on how to act in a way that benefits the community. To address this question, we might consider the service view of enhancement: since enhancements are tools to increase the competence and status of the individual, they should be judged according to how they allow for a service to the community and according to how they contribute to or detract from the ethos of service.

The service view provides a response to the problem of perverse status competition, because, as long as an ethos of service is maintained, status competition 
need not erode trust between individuals in a community. There can even be significant inequality, but when high-status persons act for the good of the community rather than for a more narrowly selfish good, then interpersonal trust can remain justified. One person's success does not come at the cost of another's defeat; on the contrary, if they are both part of the same community, the latter can benefit indirectly from the success of the former.

In what way is the service view an alternative to liberal views? First it should be emphasized that the service view does not involve a jettisoning of the principle of autonomy: there is still a meaningful distinction to be made between making one's own decisions and slavishly following what others say, do, or value. The service view is not an illiberal view. However, it is also not a liberal view. It does not endorse the dichotomy between private and public spheres: ${ }^{61}$ the ethos of service, as the set of prestige-for-service social norms, is situated in between the private and the public spheres. Neither are individuals conceptualized as self-determining agents: they are members of a community, and their ends may be significantly determined by the community they inhabit.

Is the service view a form of communitarianism? Communitarianism is understood in many different ways, and a full account is not possible here. Howeverif communitarianism implies that cultural traditions have intrinsic value, ${ }^{62}$ then the service view is not a form of communitarianism. Tradition is of value insofar it possesses timetested norms which may promote an ethos of service. However, tradition does not possess an intrinsic value that can take precedence over the needs of service. Another way of understanding the distinction is that the ethos of service defines a community through shared future rather than through a shared cultural ancestry.

A second position often associated with communitarianism is that the community is considered as a subject with its own rights. ${ }^{63}$ By contrast, in the service view, the only real agency involved here is that of individuals: the ethos of service is a guiding principle for individual decision-making, and ultimately directed towards individual well-being. Paradoxically, the service view can be thought of a refinement of the fundamental values of liberalism - but refined to such a point that the principle of autonomy ceases to be useful. Service is a principle for individuals making decisions about their life course, where decisions are made on the basis of how one can contribute to the community. Service is not antithetical to individual well-being: in reality, one achieves well-being 
precisely by being meaningful to other people. If well-being is the satisfaction of interests or preferences, then the principle of service allows one to discern what interests or preferences are worth satisfying. ${ }^{64}$

Enhancement Ethics and Science. How should one, after all this, understand the role that cultural evolutionary theory has played in the argument presented here? My suggestion is that evolutionary science should not be thought of as preempting ethical reflection, but rather as presenting the ethicist with a wealth of information about the human condition, which can inform ethical reasoning. Cultural evolutionary theory allows the ethicists a better and more detailed understanding of some of the more subtle consequences of decisions to enhance, based on facts of our status-oriented moral psychology. Humans face anxieties, worries, impostor syndromes, insecurities, competitive urges and so on - all challenges that seem crucial concerning decisions to enhance.

By arguing that a properly updated evolutionary perspective does not support liberal views, I did not argue that they are wrong or false. Rather, the implication is that liberal views, whether in social liberal or libertarian guise, just do not seem to offer meaningful guidance for the challenges that are inherent to being an individual who is also a member of a community. The challenges of anxieties, insecurities, competitiveness, and so on, would simply be categorized as obstacles to autonomy that the rational individual should ignore or avoid as much as possible.

The option is still open for the liberal to simply repudiate our evolved moral psychology, and claim it needs to be overcome in some wholesale way. For instance, liberal could still maintain that the response to the challenging psychological realities lies in more enhancement - even if that ultimately will lead us away from liberalism and towards transhumanism, as explicitly endorsed by Yuval Harari. ${ }^{65}$ However, such a strategy would only serve to underline the main thesis of this article, namely, that the evolutionary perspective on enhancement does not support a liberal view (and not an illiberal view for that matter).

Interestingly, common sense (in the form of "public opinion") seems to follow what one would expect given cultural evolutionary theory. In a recent survey study, many participants judged the ethical acceptability of enhancement to depend on features in the social environment. For instance, if enhancement is used to intensify competition, it is 
more likely to face disapproval, but if an enhancement technology is commonly used by peers or approved of by authority figures, then it is more likely to be positively evaluated. ${ }^{66}$ Enhancement use by professionals such as surgeons or pilots meets more approval than does such use by students. Elsewhere I argue in more detail that these judgments are what one would expect if one takes our status-related motivations into consideration, and given that our judgments are guided by an ethos of service. ${ }^{67}$ Thus the service view, far from repudiating our evolved moral psychology as "irrational," shows how there is an the ethical logic inherent to the service-for-prestige norms that have evolved throughout human history.

Enthusiasm or Caution? Proponents of liberal views of enhancement have often also been promotors of enhancement as such. ${ }^{68}$ Hence, in targeting liberal views, the service view will inevitably give the impression of being cautionary. The cautionary argument from service emphasizes that important classes of enhancement, including cognitive enhancements, can be used to increase dominance, and even if the enhancement does not cause any direct harm to others, over a longer period it can erode the ethos of service and lead to distrust. This cautionary argument does not underwrite an argument for prohibition, but for (moral) education, where service-for-prestige norms are communicated as ethical norms. Here the closest parallel lies with how professional bodies are supposed to self-regulate: through education and an adherence to a code of ethics, trust between professionals (and their clients) is maintained.

Nonetheless, just as a liberal view can recommend caution if an enhancement threatens to erode individual autonomy, ${ }^{69}$ the service view could also support enthusiasm. If a proposed enhancement allows an individual to provide a better service to others, and does not lead that person to assume a position of dominance over others, then it is ethically commendable. Safety considerations are relevant, of course, but even a small amount of damage to the user may be outweighed by service. For instance, even if using amphetamines causes slight neuronal damage to pilots or surgeons, it could be ethically justified if it allows them to suppress sleep and to better serve their passengers or patients can thus be ethically approved. Obviously, there is a balance to be struck: taking a lethal enhancement to provide one act of service does not make for better service over a longer time-scale. In this way, the service view on enhancement can ground an alternative way of ethically deliberating about enhancement: one that is in principle 
neither more enthusiastic or cautionary than the liberal view, but more grounded in what we know about both how humans originated, and about humans' actual desires for and experiences of enhancement.

\section{Conclusion}

How autonomous are our Millian experiments of living? To what extent are these driven by the community we inhabit, with its norms, values, and patterns of behavior? In this article, I drew on cultural evolutionary theory to show how the Darwinian competition for status has shaped our moral psychology, and a fortiori our decisions to enhance. The threat of perverse competition not only undermines evolutionary arguments for the liberal view on enhancement, but the very appropriateness of the liberal view. The principles of no-harm and of distributive justice are of limited help when faced with types of enhancement like cognitive enhancement which are positional social goods, and where the ethics (e.g., intention, orientation towards service) of the enhancer determines the ethics of the enhancement. By contrast, the service view on enhancement explicitly acknowledges the challenges of living in community, and has more in common with professional ethics rather than liberal political philosophy. Individuals in the West have been strongly influenced by the liberal focus on autonomy, but given our evolved moral psychology and underreported but clearly documented competition for status in liberal societies, the service view on enhancement appears to be an attractive alternative to the dominant liberal view.

\section{Acknowledgements}

I would like to thank Erik Parens and Greg Kaebnick for useful feedback and for helpful conversations about enhancement, and Julian Savulescu and three anonymous referees for further feedback.

\footnotetext{
${ }^{1}$ E.g. J. Harris, Enhancing Evolution: The Ethical Case for Making Better People (Princeton, NJ: Princeton University Press, 2007); L. Kass, "Beyond Therapy: Biotechnology and the Pursuit of Human Improvement," President's Council on Bioethics, Washington, DC, 2003; M. J. Sandel, The Case against
} 
Perfection: Ethics in the Age of Genetic Engineering (Cambridge, Mass: Belknap Press of Harvard University Press, 2007).

${ }^{2}$ K. L. Syme and E. H. Hagen, "Mental Health Is Biological Health: Why Tackling 'Diseases of the Mind' Is an Imperative for Biological Anthropology in the 21st Century," Yearbook of Physical Anthropology 171, no. S70 (2020): 87-117.

3 I. Persson and J. Savulescu, Unfit for the Future: The Need for Moral Enhancement (OUP Oxford, 2012).

4 Variations on this idea present in: Harris, Enhancing Evolution; J. Savulescu, A. Sandberg, and G. Kahane, "Well-Being and Enhancement," in Enhancing Human Capacities, ed. J. Savulescu, R. ter Meulen, and G. Kahane (Oxford: Blackwell Publishing Ltd, 2014), 1-18; G. Kahane and J. Savulescu, "Normal Human Variation: Refocussing the Enhancement Debate," Bioethics 29, no. 2 (2015): 133-43; N. Bostrom and A. Sandberg, "The Wisdom of Nature: An Evolutionary Heuristic for Human Enhancement," in Philosophical Issues in Pharmaceutics, ed. D. Ho, vol. 122 (Dordrecht: Springer Netherlands, 2007), 189-219; N. Agar, Liberal Eugenics: In Defence of Human Enhancement (Malden, MA: Blackwell, 2005). 5 Notre that many if not all of these targeted authors do not explicitly endorse libertarianism and often in fact allow for individual autonomy to be constrained by the principle of distributive justice (see e.g. J. Savulescu, "Procreative Beneficence: Why We Should Select the Best Children," Bioethics 15, no. 5-6 (2001): 413-26; Harris, Enhancing Evolution, at 30-31.. However, Robert Sparrow argues that the references to distributive justice are ad-hoc and that the label of "libertarian" is justified. (R. Sparrow, "A Not-So-New EUGENICS: Harris and Savulescu on Human Enhancement," The Hastings Center Report 41, no. 1 (2011): 32-42.) For the purposes of this article, we need not be concerned with how precisely to place various authors on the spectrum of liberal views.

${ }^{6}$ G. Radick, "Darwinism and Social Darwinism," in The Cambridge History of Modern European

Thought, ed. W. Breckman and P. E. Gordon, vol. Vol.1, The Nineteenth Centiry, 2 vols., 2019, 279-3000.

7 D. L. Hull, “On Human Nature," Philosophy of Science 2 (1986): 3-13.

8 Kass, "Beyond Therapy."

9 G. Ramsey, "Human Nature in a Post-Essentialist World," Philosophy of Science 80, no. 5 (2013): 98393.

${ }^{10}$ E. Hannon and T. Lewens, Why We Disagree about Human Nature (Oxford: Oxford University Press, 2018). However, value-laden concepts of human nature might subtly live on. See G. E. Kaebnick,

Humans in Nature: The World as We Find It and the World as We Create It (New York, New York:

Oxford University Press, 2014).

${ }^{11}$ Sandel, The Case against Perfection; Harris, Enhancing Evolution.

12 D. M. Buss, Evolutionary Psychology: The New Science of the Mind, 6th Edition (New York: Routledge, 2019).

${ }_{13}$ Syme and Hagen, "Mental Health Is Biological Health."

14 Kahane and Savulescu, "Normal Human Variation," 138.

15 For direct quotes, consider for instance, N. Bostrom and T. Ord ("The Reversal Test: Eliminating Status Quo Bias in Applied Ethics," Ethics 116, no. 4 (2006): 656-79) “... [O]ur current environment is in many respects very different from that of our evolutionary ancestors ... [and] places very different demands on cognitive functioning than did an illiterate life on the savanna" (pp. 665-66). Or alternatively, J. Pugh, G. Kahane, and J. Savulescu ("Bioconservatism, Partiality, and the Human-Nature Objection to Enhancement,” The Monist 99, no. 4 (2016): 406-22): “.. the relatively contingent and arbitrary features of human nature, selected as they were blind evolutionary processes..."

${ }_{16}$ Bostrom and Ord, "The Reversal Test"; Persson and Savulescu, Unfit for the Future; Kahane and Savulescu, "Normal Human Variation"; Pugh, Kahane, and Savulescu, "Bioconservatism, Partiality, and the Human-Nature Objection to Enhancement"; B. D. Earp and J. Savulescu, Love Is the Drug: The Chemical Future of Our Relationships (Manchester: Manchester University Press, 2020).

17 Bostrom and Ord, "The Reversal Test"; L. Caviola et al., "Cognitive Biases Can Affect Moral Intuitions about Cognitive Enhancement," Frontiers in Systems Neuroscience 8 (2014): 1-5.

${ }_{18}$ N. Urbinati and S. Lukes, Condorcet: Political Writings (Cambridge, UK: Cambridge University Press, 2012), at 70.

19 E. Parens, Shaping Our Selves: On Technology, Flourishing, and a Habit of Thinking (Oxford : New

York: Oxford University Press, 2015).

${ }^{20}$ Y. N. Harari, Homo Deus: A Brief History of Tomorrow (New York: Vintage, 2017). 
${ }^{21}$ David Buller distinguishes between "evolutionary psychology", the scientific theory, and "Evolutionary Psychology", the representation in wider academic circles and popular media. While the former makes narrow, testable predictions about certain cognitive process (e.g. about mate preferences, sexual taboos, or emotions as disgust: consult Buss, Evolutionary Psychology, the latter makes sweeping statements about all human traits. See D. J. Buller, Adapting Minds: Evolutionary Psychology and the Persistent Quest for Human Nature (Cambridge, MA: MIT Press, 2005), at 881.

${ }^{22}$ Savulescu, Sandberg, and Kahane, "Well-Being and Enhancement," 7.

23 E. Herrmann et al., "Humans Have Evolved Specialized Skills of Social Cognition: The Cultural Intelligence Hypothesis," Science 317, no. 5843 (2007): 1360-66.

${ }_{24}$ A seminal work in the development of cultural evolutionary theory is: R. Boyd and P. Richerson, Culture and the Evolutionary Process (Chicago: The University of Chicago Press, 1985).

25 K. Sterelny, The Evolved Apprentice (Cambridge, MA: MIT Press, 2012).

${ }^{26}$ J. Henrich, The Secret of Our Success: How Culture Is Driving Human Evolution, Domesticating Our Species, and Making Us Smarter (Princeton: Princeton University Press, 2016).

27 L. Ellis, "Dominance and Reproductive Success among Nonhuman Animals: A Cross-Species Comparison," Ethology and Sociobiology 16, no. 4 (1995): 257-333.

${ }^{28}$ J. Henrich and F. J. Gil-White, "The Evolution of Prestige: Freely Conferred Deference as a Mechanism for Enhancing the Benefits of Cultural Transmission," Evolution and Human Behavior 22, no. 3 (2001): $165-96$.

${ }_{29}$ M. van Vugt and J. M. Tybur, "The Evolutionary Foundations of Status Hierarchy," in The Handbook of Evolutionary Psychology, ed. D. M. Buss (Hoboken, NJ, USA: John Wiley \& Sons, Inc., 2015), p. 802.

$3^{\circ}$ M. E. Price and M. Van Vugt, "The Evolution of Leader-Follower Reciprocity: The Theory of Service-forPrestige," Frontiers in Human Neuroscience 8 (2014): 1-17.

${ }^{31}$ Van Vugt and Tybur, "The Evolutionary Foundations of Status Hierarchy."

32 Except if, through the vagaries of fashion or the contingency of cultural evolution, the useless

enhancement becomes a status signal. The custom of foot binding seems to be an example of this: foot binding is an "enhancement" in the sense of keeping adult foot size abnormally small, and despite coming at a terrible price, it nonetheless spread in historical Chinese societies in large part because it had become a status signal.

33 L. Ellis, "The High and the Mighty among Man and Beast: How Universal Is the Relationship between Height (or Body Size) and Social Status?," in Social Stratification and Socioeconomic Inequality: Vol. 2: Reproductive and Interpersonal Aspects of Dominance and Status, ed. L. Ellis (Westport, CT: Praeger, 1994), 94-111.

34 Van Vugt and Tybur, "The Evolutionary Foundations of Status Hierarchy"; N. M. Blaker et al., "The Height Leadership Advantage in Men and Women: Testing Evolutionary Psychology Predictions about the Perceptions of Tall Leaders," Group Processes \& Intergroup Relations 16, no. 1 (2013): 17-27.

35 D. B. Allen, "Growth Promotion Ethics and the Challenge to Resist Cosmetic Endocrinology," Hormone Research in Paediatrics 87, no. 3 (2017): 145-52, at 146.

${ }^{36}$ R. H. Steckel, "Stature and the Standard of Living," Journal of Economic Literature 33, no. 4 (1995): 1903-40.

37 D. Redhead, J. Cheng, and R. O'Gorman, "Status Competition and Peer Relationships in Childhood," in Encyclopedia of Evolutionary Psychological Science, ed. T. K. Shackelford and V. A. Weekes-Shackelford (Cham: Springer International Publishing, 2018), 1-9.

$3^{8} \mathrm{~J}$. Kegelaers et al., "Incentives and Deterrents for Drug-Taking Behaviour in Elite Sports: A Holistic and Developmental Approach,” European Sport Management Quarterly 18, no. 1 (2018): 112-32.

39 S. Wright, S. Grogan, and G. Hunter, "Motivations for Anabolic Steroid Use Among Bodybuilders," Journal of Health Psychology 5, no. 4 (2000): 566-71.

40 Buss, Evolutionary Psychology.

${ }^{41}$ C. Anderson, J. A. D. Hildreth, and L. Howland, "Is the Desire for Status a Fundamental Human Motive? A Review of the Empirical Literature.," Psychological Bulletin 141, no. 3 (2015): 574-601.

42 E. Juengst and D. Moseley, "Human Enhancement," in The Stanford Encyclopedia of Philosophy, ed. E. N. Zalta, (2019).

43 Earp and Savulescu, Love Is the Drug.

44 A. Buchanan and R. Powell, The Evolution of Moral Progress: A Biocultural Theory (Oxford, New York: Oxford University Press, 2018); I. Persson and J. Savulescu, "The Evolution of Moral Progress and Biomedical Moral Enhancement," Bioethics 33, no. 7 (2019): 814-19. 
45 Persson and Savulescu, "The Evolution of Moral Progress and Biomedical Moral Enhancement," 818. ${ }^{46}$ M. J. Mehlman and J. R. Botkin, Access to the Genome: The Challenge to Equality (Washington, D.C: Georgetown University Press, 1998); A. Buchanan et al., From Chance to Choice: Genetics and Justice (Cambridge, UK: Cambridge University Press, 2001); A. Buchanan, "Enhancement and the Ethics of Development," Kennedy Institute of Ethics Journal 18, no. 1 (2008): 1-34.

47 Buchanan and Powell, The Evolution of Moral Progress.

48 J. Rawls, A Theory of Justice, Revised edition (1971; repr., Cambridge, MA: The Belknap Press of Harvard University Press, 1999), at 53.

49 J. Savulescu, B. Foddy, and M. Clayton, "Why We Should Allow Performance Enhancing Drugs in Sport," British Journal of Sports Medicine 38, no. 6 (2004): 666-70.

$5^{0}$ Kegelaers et al., "Incentives and Deterrents for Drug-Taking Behaviour in Elite Sports."

${ }_{51}$ This can, for instance, read into the importance placed on the notion of a "level playing field". See for instance B. Kayser, A. Mauron, and A. Miah, "Current Anti-Doping Policy: A Critical Appraisal," BMC Medical Ethics 8, no. 2 (2007).

$5^{2}$ See for instance H. Greely et al., "Towards Responsible Use of Cognitive-Enhancing Drugs by the Healthy," Nature 456, no. 7223 (2008): 702-5.

53 A. Abbott, The System of Professions: An Essay on the Division of Expert Labor (Chicago and London: University of Chicago Press, 1988).

54 Anderson, Hildreth, and Howland, "Is the Desire for Status a Fundamental Human Motive?"

55 R. G. Wilkinson, Mind the Gap: Hierarchies, Health and Human Evolution (New Haven and London: Yale University Press, 2001); M. Marmot, Status Syndrome: How Your Social Standing Directly Affects Your Health (London: A\&C Black, 2005).

${ }^{5}$ K. L. Fingerman et al., "Helicopter Parents and Landing Pad Kids: Intense Parental Support of Grown Children," Journal of Marriage and Family 74, no. 4 (2012): 880-96.

57 C. Segrin et al., "Parent and Child Traits Associated with Overparenting," Journal of Social and Clinical Psychology 32, no. 6 (2013): 569-95.

${ }_{5} 8$ M. del M. Salinas-Jiménez, J. Artés, and J. Salinas-Jiménez, "Education as a Positional Good: A Life Satisfaction Approach,” Social Indicators Research 103, no. 3 (2011): 409-26.

59 For an argument applied to professionalism in scientists, see H. Desmond, "Incentivizing Replication Is Insufficient to Safeguard Default Trust," Philosophy of Science, no. 88 (2021): 1-12.

${ }^{60}$ E. Freidson, Professionalism, the Third Logic: On the Practice of Knowledge (University of Chicago Press, 2001); H. Desmond, "Professionalism in Science: Competence, Autonomy, and Service," Science and Engineering Ethics 26, no. 3 (2020): 1287-1313.

61 Buchanan et al., From Chance to Choice, at 378-80.

62 D. Bell, "Communitarianism," in Stanford Encyclopedia of Philosophy, ed. E. N. Zalta, 2020.

63 C. E. Baker, "Sandel on Rawls," University of Pennsylvania Law Review 133, no. 4 (1985): 895-928.

64 The distinction between more and less valuable preferences has remained a difficult challenge for preference utilitarianism. See for example, M. C. Nussbaum, "Beyond 'Compassion and Humanity': Justice for Nonhuman Animals," in Animal Rights: Current Debates and New Directions, ed. C. R. Sunstein and M. C. Nussbaum (Oxford: Oxford University Press, 2004), 299-320.

65 Harari, Homo Deus.

${ }^{66}$ C. T. Dinh, S. Humphries, and A. Chatterjee, "Public Opinion on Cognitive Enhancement Varies across Different Situations," AJOB Neuroscience 11, no. 4 (2020): 224-37.

${ }^{67}$ H. Desmond, "Service and Status Competition May Help Explain Perceived Ethical Acceptability," AJOB Neuroscience 11, no. 4 (2020): 258-60.

68 Parens, Shaping Our Selves.

${ }^{69}$ For an example, see J. Harris and S. Chan, "Moral Behavior Is Not What It Seems," Proceedings of the National Academy of Sciences 107, no. 50 (2010): E183-E183. 